\title{
Exploration of Gold Colloidal beyond Trillion Times Dilution
}

Partha Kumbhakar ${ }^{1}$, Kumaravel. V ${ }^{2}$, Ashit Kumar Pramanik ${ }^{3}$, Rajesh Chaudhary ${ }^{2}$, S Prasanth ${ }^{2}$, Julka Kumari Tripathi ${ }^{2}$, Amreesh Chandra ${ }^{4}$, Himashu Sekhar Tiwary ${ }^{5}$, Subash Singh ${ }^{2}$, Chandra Sekhar Tiwary ${ }^{1 *}$

${ }^{1}$ Department of Metallurgical and Materials Engineering, Indian Institute of Technology

Kharagpur, Kharagpur 721302, India

${ }^{2}$ National Institute of Homoeopathy, Kolkata,700106, India.

${ }^{3}$ National Metallurgical laboratory, Jamshedpur, India, 831039

${ }^{4}$ Department of Physics, Indian Institute of Technology Kharagpur, Kharagpur 721302, India ${ }^{5}$ Central Government Health Scheme, New Delhi, India.

KEYWORDS: Nanoparticles; Colloidal solution; Optical property; Homoeopathy; Top and bottom distribution of NPs; 


\section{ABSTRACT}

The physical and chemical properties of the colloidal solution change as a function of the concentration of nanoparticles. Here, we have studied the effect of systematic ultra-low dilution (up to $10^{200}$ times) of gold nanoparticle colloidal, revealing the mechanism of colloidal formation in such extreme dilution. Optical spectroscopy confirms the presence of nanoparticles with narrow size distribution and high surface activity in higher dilutions (up to $10^{200}$ times) colloidal solution. Particle size analysis of the serially diluted solution shows that the size distribution becomes morenarrow with increasing dilution. Transmission electron microscopy confirm the presence of nanoparticle up to $10^{200}$ times dilution. The process of dilution introduces an active carbon layer on these particles. The understanding of such extreme dilution can be utilized in biomedical applications, especially in the Homoeopathy system. 


\section{INTRODUCTION}

Colloidal solution of metals has been utilized for energy, water treatment, catalysis, sensor, biomedical, and many other applications. ${ }^{[1-5]}$ In most of these cases, a large amount of emphasis has been given to processes to increase composition/concentration of metallic nanoparticles in the colloidal. There is limited study on the process of dilution (1bpm or ultra-dilution beyond $\left(10^{15}\right)$ and its effect on different physical and chemical properties. Since 1796, a highly diluted colloidal solution is used for medication in Homeopathy. The various literature of Homeopathy medicines suggest that colloidal solutions are more effective with higher potentization/dilution (composed of serial dilutions and vigorous triturations/successions beyond Avogadro no- $10^{23}$ ). Multiple studies about homeopathic potencies and nanoparticles (NPs) have been published ${ }^{[6-17]}$. The study published by Chikramane et al. ${ }^{[6,8]}$, showing the presence of starting material in the form of nanoparticles, has been the most pivotal. Following this study, multiple advancements have been made further to understand the complex interactions of homeopathic medicine. Basu et al. ${ }^{\text {[7] }}$ reported the physico-chemical changes in nanoparticles due to Homeopathic trituration and succussions. Wassenhoven et al. ${ }^{[9]}$ performed nanoparticle characterization of homeopathic medicines and showed the nanoparticle-induced hormetic activation ${ }^{[8]}$. Bhattacharyya et al. ${ }^{[10]}$ demonstrated the nanoparticles enhance cellular uptake and increase bioactivity. Many groups are still working to explain the origin of nanoparticles while processing. The change in size and morphology of the nanoparticles during different potencies is not explained. Also, the increase in the effectiveness of the medicine in higher potency is not clear.

In the current work, we have processed a serial dilution of Aurum Met (gold nanoparticle colloidal) by a conventional method. We have chosen different tools (spectroscopy and microscopy) to understand the effect of serial dilution and succussions. Spectroscopy measurement 
shows that the solution contains Au nanoparticles with different size distributions. Electron microscopy has been used to confirm the presence of Au NPs in different morphology and size. The microscopy analysis reveals the mechanism of the formation of the nanoparticles. Particle size and zeta potential also measure to know the effective surface charge of the nanoparticles.

\section{EXPERIMENTAL SECTION}

\subsection{Synthesis of Sample:}

The Gold with $99.9 \%$ purity was used for the preparation of Aurum Met. The sample was prepared in a glass vial as well as in a plastic vial following the standard protocol of Homoeopathic Pharmacopeia, India ${ }^{[16-17]}$. The sample and procedure were performed in the HAPCO Pharmaceutical laboratory. The detailed processing step is described in supporting information. A schematic representation of the process steps is shown in Figure 1.

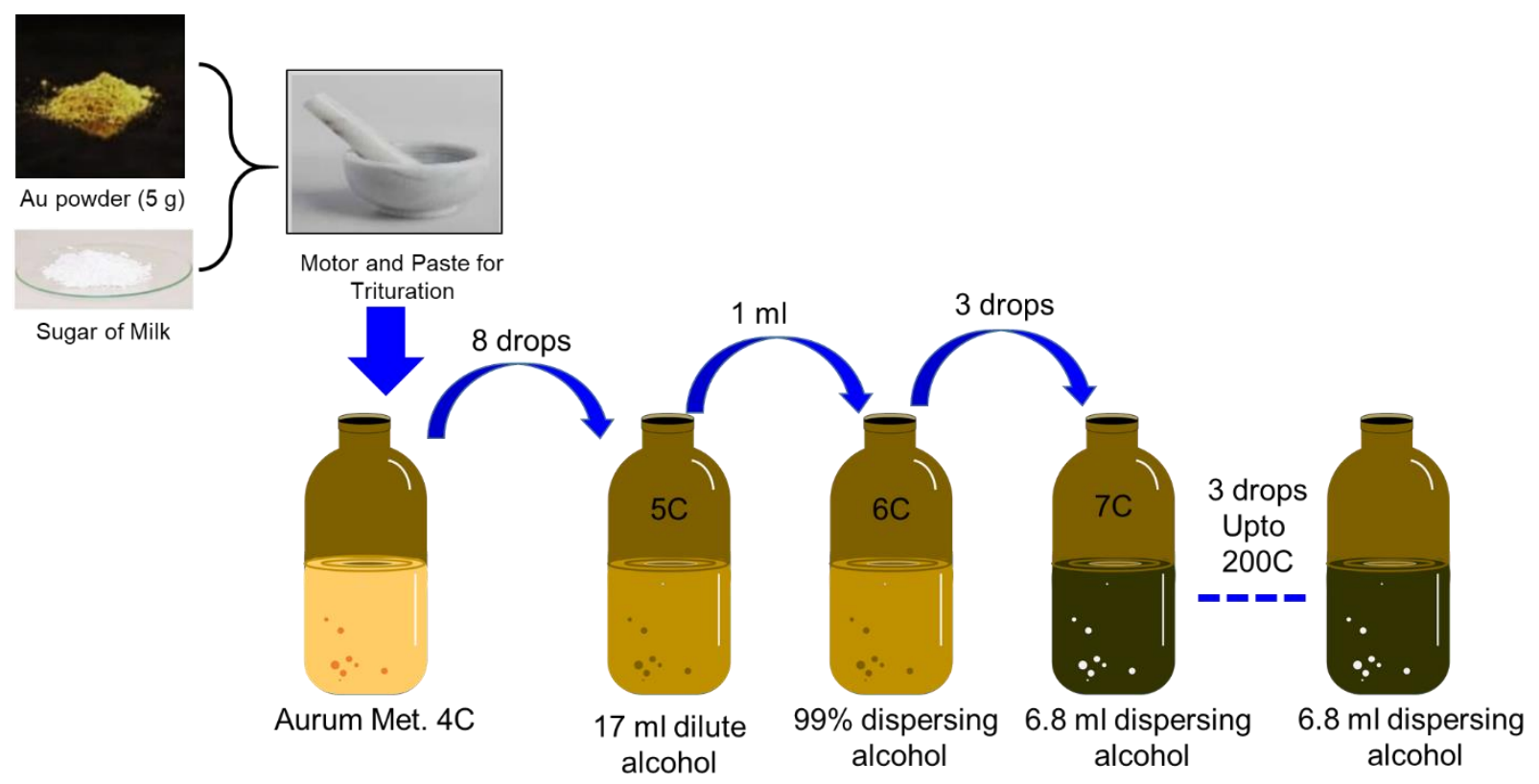

Figure 1. Schematic diagram of the dilution process of Au NPs 
Characterizations: The structural analysis of the power sample ( $3 \mathrm{X}$ and $6 \mathrm{X})$ was carried out using a PANalytical X'Pert diffractometer (XRD) with $\mathrm{Cu}-\mathrm{K} \alpha$ incident radiation $(\lambda=0.15406 \mathrm{~nm})$ in the scan range of $10^{\circ}$ to $90^{\circ}$ with a scan rate of $1^{\circ}$ per min. UV-visible absorption spectra are recorded with a double beam spectrophotometer. Raman spectroscopy measurements were performed by WITec UHTS Raman Spectrometer (WITec, UHTS 300 VIS, Germany) at an excitation wavelength of $532 \mathrm{~nm}$. Transmission electron microscopy (TEM- JEOL JEM 2200FS), was carried out to know the particle size in the diluted sample (24C and 200C). Zeta potential and particle size measured using Zeta analyzer and particle size analyzer (Model No: Horiba Scientific SZ-100).

\section{RESULTS AND DISCUSSION}

The initial solid powder samples (3X and $6 \mathrm{X}$ ) were characterized using XRD. The colloidal suspension samples (6C-200C) were characterized using spectroscopy and microscopy techniques.

\subsection{XRD pattern of triturated powder samples (3X and 6X)}

In order to characterize (presence of phases, size, and strain) the Gold (Au) and sugar of milk mixture during mechanical grinding, XRD analysis has been performed. Figure $2 \mathrm{~A}$ shows the XRD pattern of the powder sample with different concentrations of Au and sugar of milk. The diffraction peaks confirm the presence of Au NPs with lactose (sugar of milk) peaks. The peak at $38^{\circ}$ corresponds to the (111) plane of Au nanoparticles and matches with the standard JCPDS card

${ }^{[18]}$. Along with the Au NPs peak, we also observe other peaks corresponding to the lactose. We do not observe any other impurity peaks. 
Figure 2B shows the XRD peak of only (111) Au NPs for two different triturations (3X and $6 \mathrm{X}$ ). For higher trituration (i.e., 6X), we observe a higher angle shift with a line broadening as compared to $3 \mathrm{X}$ trituration. The shift of the XRD pattern reveals the presence of strain $(\sim 4 \%)$ in the materials. The origin of the strain is due to the mechanical grinding during trituration. Here, we have used the Debye Scherrer equation for calculating the crystalline size of the samples ${ }^{[19]}$. The $3 \mathrm{X}$ and $6 \mathrm{X}$ triturated sample shows a crystalline size of $\sim 2.6$ and $2.4 \mathrm{~nm}$ respectively. The reduction in crystalline size is due to continuous mechanical grinding of metallic Gold in the presence of covalently bonded sugar of milk crystals. The mechanical grinding results in lattice strain, and after critical stress, the Au particles fracture and form nano sized crystalline materials. It is important to note that, as per previous observations, ${ }^{[6,20]}$ NPs are found in higher dilution medicine samples, but the origin of nanoparticle formation is not reported so far. Based on the current observation, we can clearly see that the nanocrystalline Au NPs are formed during the trituration process itself. This highlights a unique observation that the initial preparation (starting material) for the high homeopathic dilution is very much different from the other conventional medicinal preparation, which was never noticed so far, leading to erroneous conclusions.
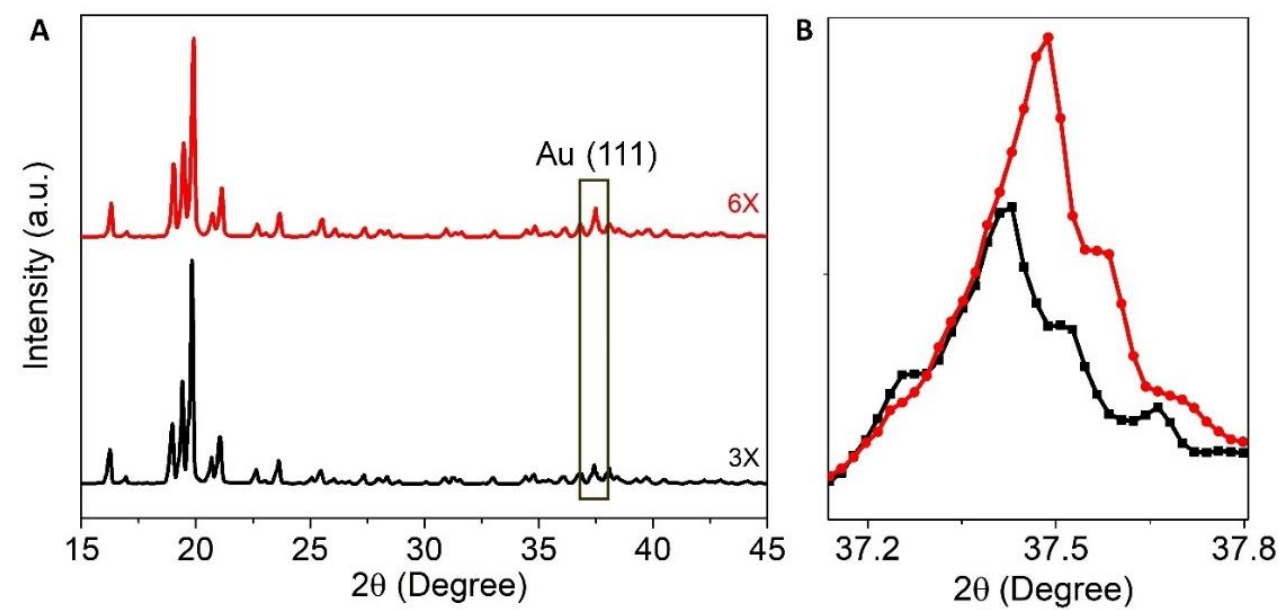

Figure 2. A. XRD pattern of power sample with two different grindings. B XRD peak of Au NPs. 


\subsection{UV-vis absorption spectra of liquid colloidal suspension samples (all diluted samples: 6C-200C)}

The UV-vis absorption spectroscopy measurement is an essential tool, and it can provide helpful information about the presence of materials in the homeopathic potencies (different dilutions). Figure 3A shows a schematic diagram of a glass bottle containing the homeopathic potency. The spectra of the solution have been taken from two different portions of the glass bottle (upper and lower part), as shown in Figure 3A. Figure 3B-C shows the variation of UV-Vis absorption spectra of the top and bottom part solution with different potencies. The presence of three absorption-band is observed for all samples. To know the exact positions of the bands, we have plotted the second derivative of a spectrum as a function of wavelength (Figure 3D). The occurrence of absorption band at $\sim 557 \mathrm{~nm}$ and $\sim 721 \mathrm{~nm}$ due to the presence of anisotropic structure

of different sized Au nanoparticles with localized surface plasmon resonance (LSPR). ${ }^{[3,21]}$ Additionally, it also shows that the shape and absorption intensity of the spectra change with potencies. At higher potency (i.e., concentration- $10^{6}$ to $10^{17}$ times dilution), a broad absorption band has been observed, and it gradually became narrow/sharp after 24C. Similar variation has been observed for both the upper and lower part of the potencies.

The obtained absorption spectra have been fitted by the Gaussian multiple peak fitting method to know the presence of NPs in the potencies (Figure S1). A representative Gaussian fitted plot of 6C potency is shown in Figure 3E, and other diluted samples are shown in supporting figures. From the fitting, we have observed that three major peaks are present. The first peak, at $\sim 456 \mathrm{~nm}$, is associated with silica NPs. The peak is absent in samples processed in plastic bottles; the details are described in supporting information (Figure S2). The other two peaks originate due to the localized surface plasmon resonance (SPR) of Au NPs. The narrowing of the absorption peaks in 
the absorption spectra (Figure 3A and 3B) of the samples indicates the relatively narrow size distribution of the Au NPs. To know the exact origin of the narrowing of the Au absorption edge, we have plotted the full width half maximum (FWHM) as a function of potencies, as shown in Figure 3F. The FWHM decreases with an increase of potencies, which means the deviation in Au nanoparticle size distribution decreases in higher potency. The distribution of particle size is different for the top and bottom parts of the potency.
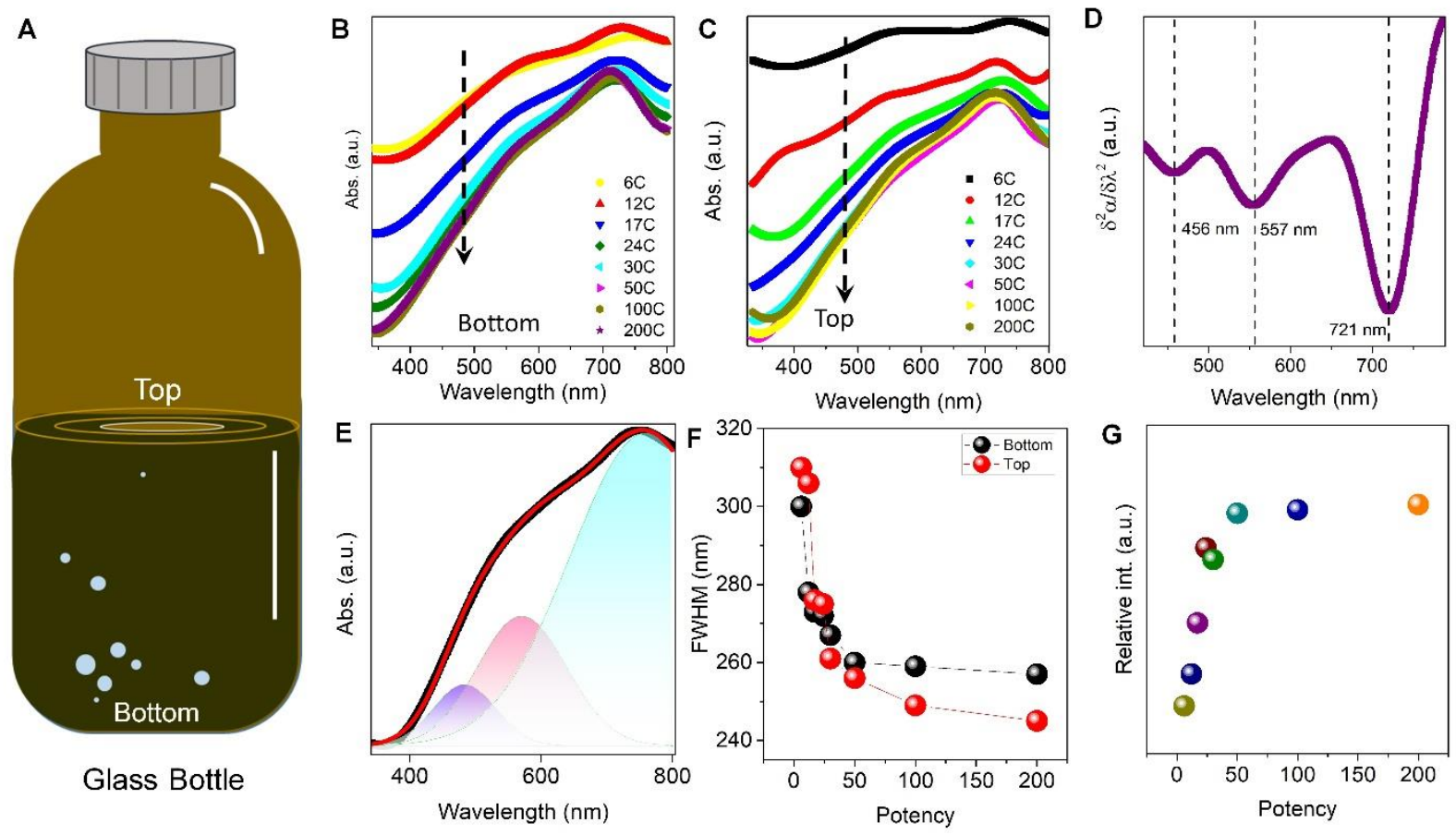

Figure 3. A. Schematic presentation of a sample bottle with liquid dispersion. UV-Vis absorption spectra of the sample with different dilutions. Samples are taken from the bottom (B)and bottom and top (C) parts of the glass bottle. (D) Second-order derivative of the absorption spectra as a function of wavelength. Indicates the presence of LSPR and different oscillation modes of Au nanoparticles. (E) Baseline corrected fitted absorption spectra with three Gaussian peaks of 200C sample. (F) Variation of FWHM as a function of dilution at two different portions of the bottle. (G) Variation of the relative intensity of absorbance of Au NPs. 
Also, the Au NPs size variation is different for the top and button part of the potency, as shown in Figure 3F. The Au NPs peak becomes more prominent in the higher potency samples, as shown in Figure 3G. Hence, we can conclude that as the potency increases, the ratio of Au nanoparticle to lactose increases. The three critical observations in current characterization are:

A. The distribution of particles is not uniform across the dilution (top and bottom parts are different).

B. The distribution of the particles changes as a function of potency.

C. the ratio of $\mathrm{Au} /$ sugar increases as an effect of homeopathic potentization.

The succussion process was studied in detail using optical measurements. The UV-vis absorption spectra have been taken in different successions for different potencies. A representative spectrum of 200C potency after different succussions is shown in Figure 4A; other spectra are shown in supporting information (Figure S3). Figure 4A(I) shows an enlarged view of the first absorption band ( $210-260 \mathrm{~nm})$, denoted as I. This peak is due to the presence of carbon sources $(\mathrm{C}=\mathrm{C}, \mathrm{C}=\mathrm{O}$ groups $)$ in the solution. ${ }^{[22,23]}$ The peak intensity increases with the increase of succussion. Figure 4A(II) presents the second absorption band, as denoted II, originating from the aromatic $\pi$-system. ${ }^{[23]}$ This band also shows similar nature as the succussion number increases, peak intensity increases. ${ }^{[23]}$ During the succussion process, the carbon layers disintegrate and lead to an increase in carbonyl groups. In Figure $4 \mathrm{~A}(\mathrm{III})$, we present the absorption spectra of $\mathrm{Au}$ nanoparticles as a function of succussions. The intensity of Au NPs peak increases as a function of succussion. During the succussion process, the sugar/lactose layers dissolve in the solvent (water/alcohol mixture), leading to nanoparticle exposure on the surface. Therefore, in subsequent 
succussions of potency, the fraction of active nanoparticles (nanoparticles on the surface of the sugar matrix) increases, stimulating the response of the medicines in human health. Based on the absorption spectra, the absorption intensity was calculated for different potencies $(6 \mathrm{C}, 50 \mathrm{C}$, and 200C) as a function of succussion, as shown in Figure 4B-D. It shows that the absorption intensity (defined as P1 and P2) increases with an increase of succussions. The trend remains similar for different potencies $(6 \mathrm{C}, 50 \mathrm{C}$, and $200 \mathrm{C})$.
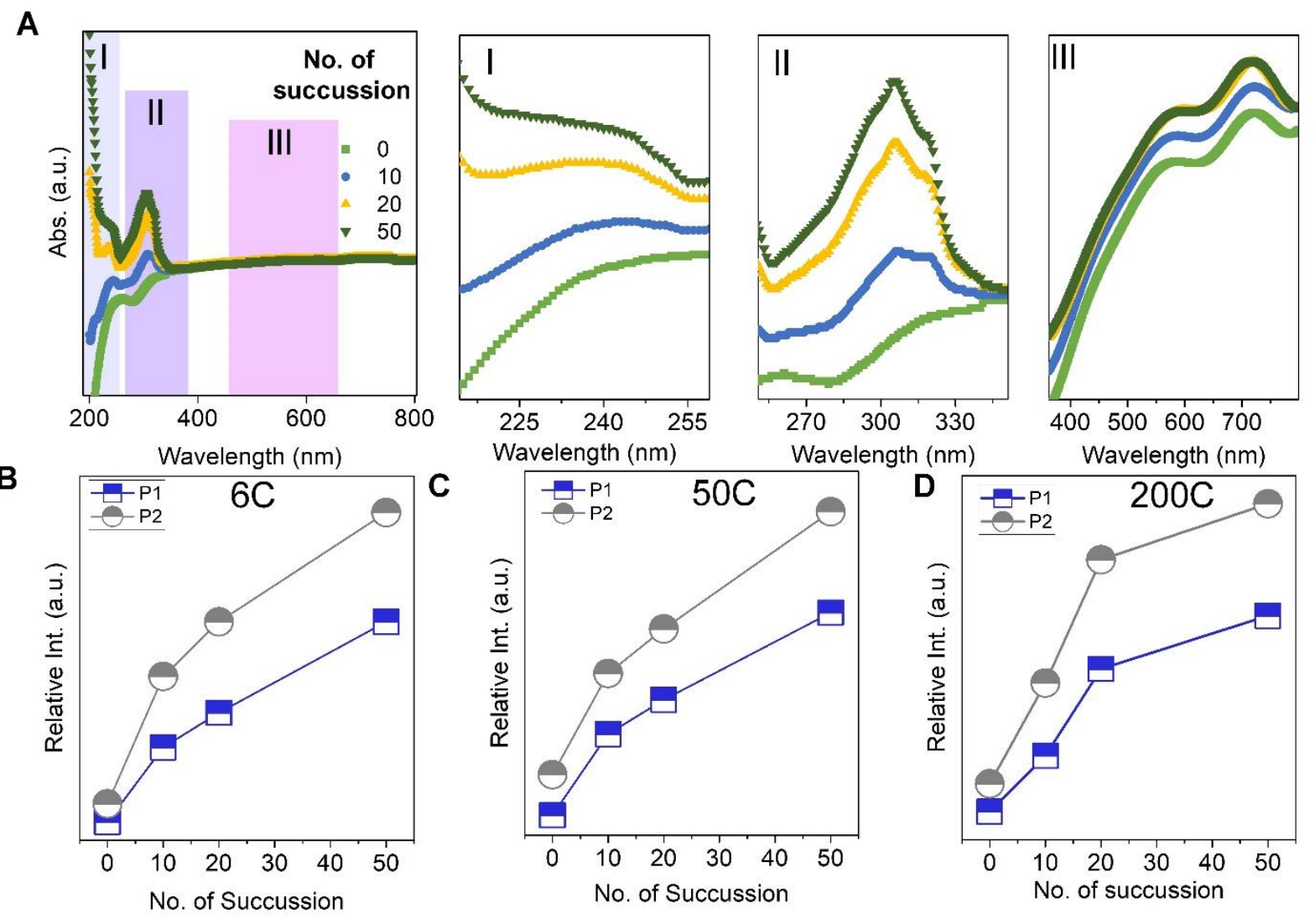

Figure 4. A UV-vis absorption spectra of the sample (200C) after and before successions. I, II, and II show the enlarged portion of the marked area. The variation of absorption intensity of Peak I (P1) and Peak II (P2) for (B) 6C, (C) 50C, and (D) 200C sample as a function of succession. 


\subsection{Particle size and zeta potential analysis}

Quantitative measurement of particles present in the different potencies is performed using DLS, as shown in Figure 5A-D. To measure particle size, we have taken the solution in two different parts of the glass bottle (top and bottom part), as described earlier. Most of the particles are in size in the upper portion of the bottle $(\sim 200-600 \mathrm{~nm})$ (Figure 5A). However, mostly large size $(\sim 6 \mu \mathrm{m})$ particles are found (Figure 5B). The distribution of particle size decreases (size range of particles present) as the potency increases. It is important to note that the bulk particle size measurement (large sample volume) using DLS clearly shows the non-uniform size distribution of particles in the solution (top and bottom part). Hence, during the potentization process, we take the solution from the top part, i.e., smaller particles. Hence, potentization results in solutions with smaller particles, which improves the effectiveness of the medicine on human health.

We have measured the particle size in different successions, as shown in Figure 5C-D (upper and lower parts of the bottle). Interestingly, we have seen that in the upper part of the bottle, the average size of the particles decreases with an increase of successions number. Hence, more active nanoparticles are exposed to the surface. However, in the lower part, the size of the particle increases with succession during potentization. This result indicates an increase in the air-bubble formation and a mixture of nanoparticles with bubbles.

Zeta potential is also an essential parameter for knowing the stability of colloidal systems. It also helps to quantify the surface charge of the particle. Here, the zeta potential of all samples has been measured using the Zeta analyzer measurement system, as shown in Figure 5E-F. The plot of zeta potential as a function of potency shows a change of the value from negative to positive, which means the nature of the solutions is very unstable. Such experimental values suggest the un- 
stability of the solution i.e., the activity of the charged particles changes succession and potentization which is consistent with our optical measurements (i.e. more active Au NPs).
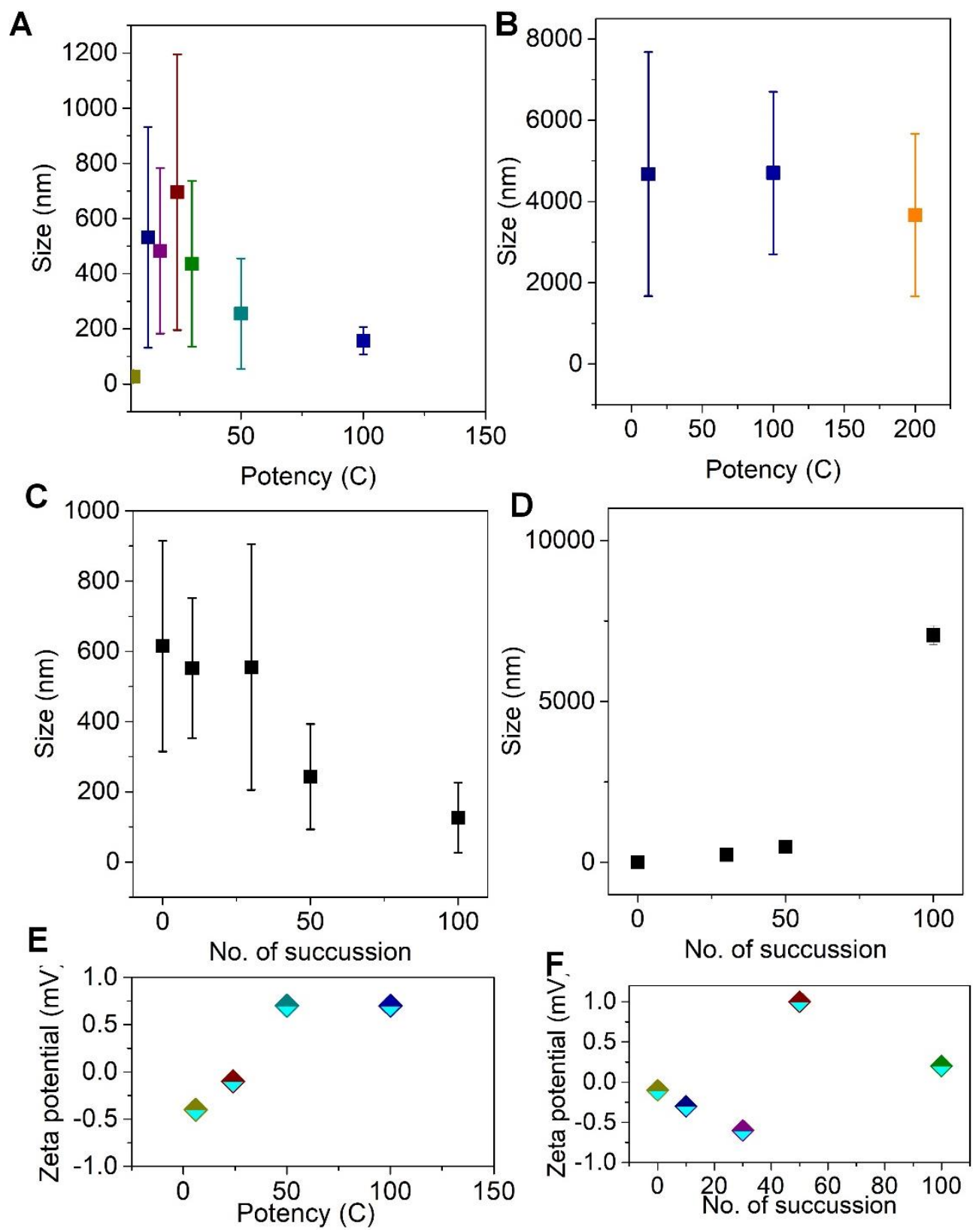

Figure 5. The variation of particle size in two different positions of the sample container (A) Top and (B) Bottom, respectively. Variation of particle size after different successions in blank solution in (C)Top and (D) bottom portion. (E) Zeta potential of the all diluted sample. (F) Variation of zeta potential with successions. 


\subsection{TEM and Raman measurement of low and high diluted sample}

The above discussions are further supported using the TEM study, as shown in Figure 6. Here, two different potencies (low and high) are used to understand the morphology (size, shape etc.) and chemical composition of the particles. Figure 6A-C shows the TEM and high-resolution TEM (HRTEM) images of a low potency sample with different size distribution. It is observed that Au NPs are embedded in the carbon layer. Figure 6B shows that the Au NPs are distributed on the carbon layer. The carbon layer is formed from the sugar of milk (sucrose, glucose, fructose etc.). The HRTEM image of Au NPs, shown in Figure 6C, confirms the well crystalline nature of the nanoparticles. However, in the case of higher potency, well-separated monodispersed Au nanoparticles are observed, as shown in Figure 6D-G. Figure 6E-F shows a single particle of Au coated with a carbon layer. Interestingly, here we have not observed any large carbon layer. HRTEM image shows (Figure 6E) that the surface of Au NPs is affected by electron beam irradiation. These results confirm that the surface of Au NPs is more reactive at a higher potency. Figure 6G confirmed the reactive edge of the Au NPs. We have schematically presented the TEM observation in Figures 6H and 6I. It is observed that a carbon layer of sugar milk is formed at low potency, and the Au NPs are embedded in the carbon layer. However, when diluted the solution, the carbon layer may disintegrate and form a carbon coating on every nanoparticle. Additionally, at very high potency, the surface of the particles is more active, and this active surface reacts with humans. We have taken the Raman spectra of different diluted samples to confirm our assumption, and obtained results are well-matched with the TEM results. As shown in Figure 6J, the Raman vibrations are well-matched with previously reported data. ${ }^{[24]}$ It is found that several Raman peaks have appeared related to sucrose, lactose, and glucose, which strongly supports the TEM results. 

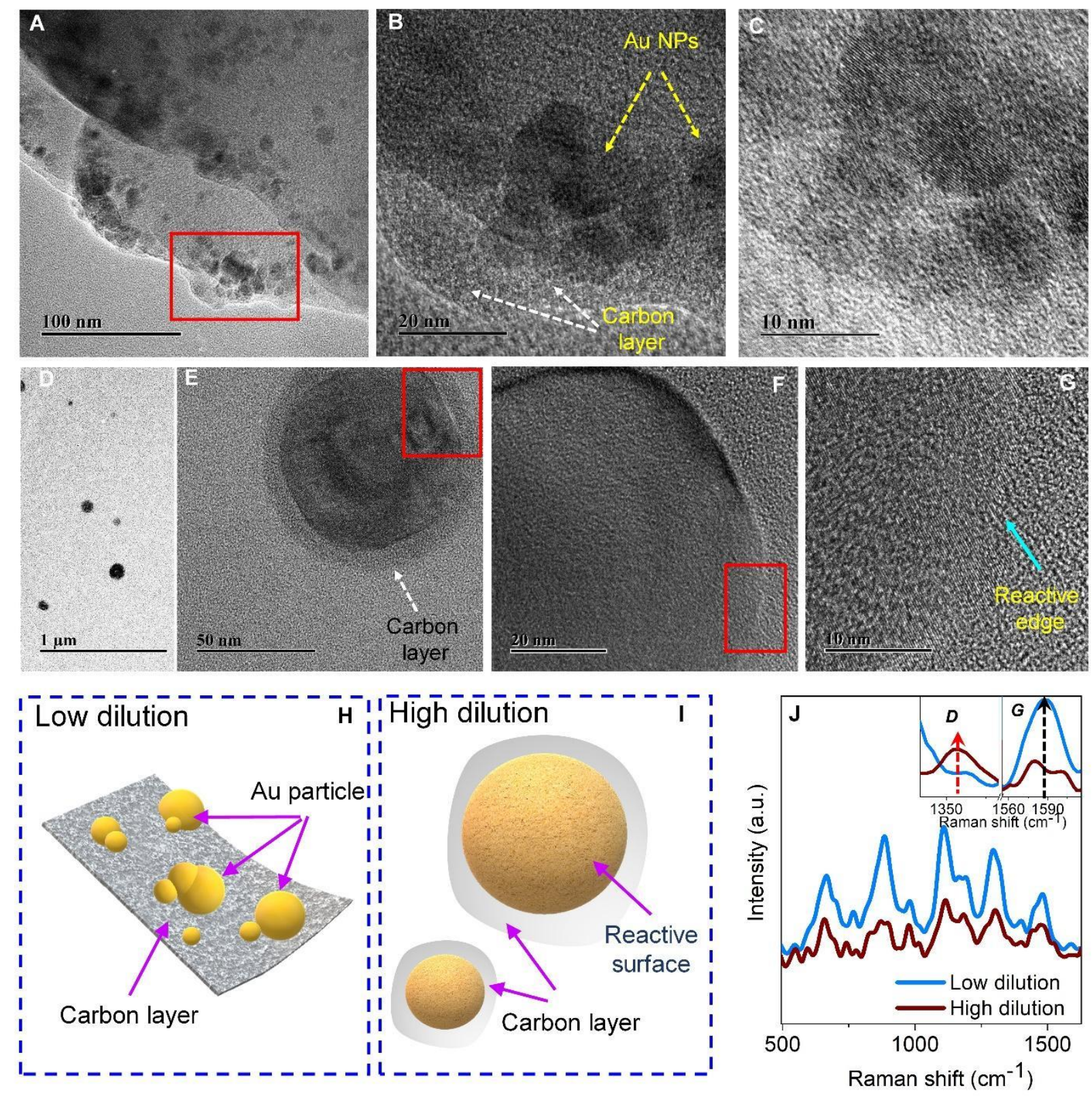

Figure 6. A TEM image of a low dilution sample. (B) HRTEM image of the sample. (C) HRTEM image of Au NPs. (D) TEM image of high diluted sample. (E) Single Au NPs with carbon layer at high dilution. (F) The enlarged portion of Figure E. (G) Reactive surface edge of Au nanoparticle. Schematic representation of $(\mathrm{H})$ low dilution and (I) high dilution sample. (J) Raman spectra of both samples. 
Additionally, it is found that the intensity of the Raman peaks varied with dilution. Previously it was reported that the Raman intensity strongly depends on the concentration of the sample when other parameters of the excitation source are constant ${ }^{[25]}$. Therefore, this is a clear indication of the effective coating of the carbon layer on Au NPs. The spectra show that lactose/fructose/glucose molecules firmly stabilize the Au NPs by making a layer over the nanoparticles.

\subsection{Tyndall effect of all diluted samples}

Digital photographs of the sample (before and after succession) of different potencies (6C, 50C, and 200C) are fully transparent. However, they show light scattering properties under laser beam excitation due to the Tyndall effect (Figure 7A-D). The Tyndall effect confirms the presence of particles in the solution. For further analysis of the presence of air-bubble-particle, line scans were performed using ImageJ software, as shown in Figure 7B-D. It has been found that some spikes are noted, which are due to the presence of particles. Inset shows the digital photographs of the samples (before and after succussions) under Laser excitation. Interestingly, after succussions, a drastic change is noted. The appearance of the new spikes indicates the formation of air-bubblesparticle mixtures. The size and shape of the spikes also changes are associated with the formation of different size floated air-bubble-particle mixtures. ${ }^{[26-27]}$ 


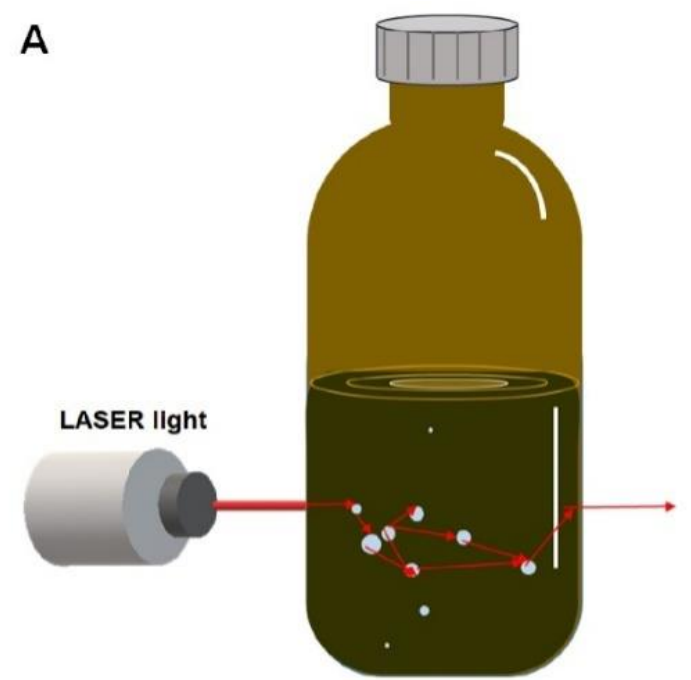

Tyndall effect
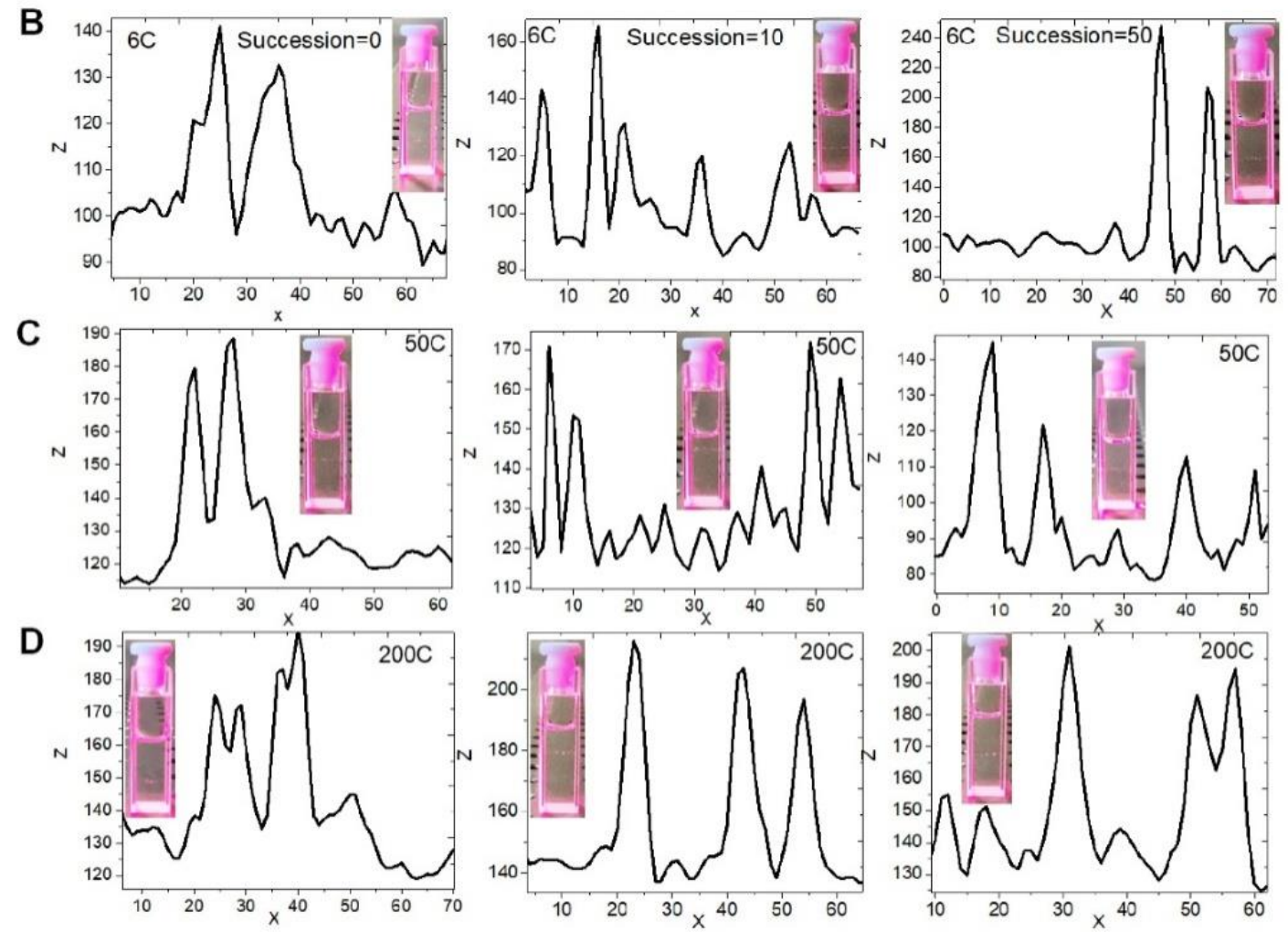

Figure 7 A. Schematic shows the Tyndall effect in a low potency sample. Digital photographs with line scan profiles of the samples before and after successions (B) 6C, (C) 50C and (D) 200C. 


\section{CONCLUSIONS}

Here, we have mixed the gold piece with the milk of sugar for the preparation of powder. The gold plate has been mechanically grinded very well by adding milk of sugar. After mechanical grinding, we got an Au particle and sugar (lactose) combination powder. XRD measurement confirms the presence of $\mathrm{Au}(\sim 2.6 \mathrm{~nm}$ crystallite size). Also, we observe a shift due to repeated mechanical grinding when we were mixing Au particles and sugar powder. After that, we mix this mixed powder with Dispensing Alcohol. We have prepared solutions in different proportions and gradually decreased the concentrations, such as $6 \mathrm{C}, 7 \mathrm{C}$ up to $200 \mathrm{C}$. The absorption characteristics of Au NPs are getting more prominent as the ratio of sugar and Au particles is gradually decreasing. The higher potentization removes the sugar layer and exposes Au NPs. With the repeated successions in the solution, the layer of sugar moves away from the top of the NPs, and the surface of the NPs is coming out. The more surface of these NPs comes out, the more quickly it will react with human health and increase its medicinal effect. At low dilution, there is a significant carbon sheet/layer and Au NPs embedding on it. However, in the case of high dilution, we observed that instead of this sheet/layer, each particle is wrapped in a sheet. Also, we have observed that the surface of the nanoparticle is more reactive. Therefore, this reactive surface of each particle can easily attach to the human organ and quickly cure diseases. Raman analysis shows that the D band and $\mathrm{G}$ band of carbon have been created, and their intensity has changed with dilution. Therefore, we can say that when we do more and more dilution, the properties of nanoparticles become more prominent and the less the dominance of lactose. Simultaneously, when we shake it repeatedly, the layer of sugar is removed from the surface of the NPs, and the NPs are exposed; thus, the nanoparticles are more capable of working in human health. Tindal effect shows the particles in the solutions, and the number of these particles increases with shaking. The reason for the increase 
in particle size is that we can see that more and more water bubbles are forming as we go. This is why homeopathic medicines need to be shaken repeatedly before taking them to make them more effective. We may conclude that higher potencies of Aurum Met expressing the dynamic action in the presence of Nanoparticles. In the drag-delivery system, the agglomeration and clustering of nanoparticles have a wide range of applications. In homeopathy medicine, these agglomeration and clustering have an enormous effect due to the presence of nanoparticles. In higher dilution, the nanoparticle surface energy, particle-particle interaction, ionic strength, uniform distribution etc. determine the effectiveness in several applications. Therefore, we believe that this work provided a new pathway for future applications in the drug delivery system. 


\title{
ASSOCIATED CONTENT
}

\section{Supporting Information}

Synthesis of Sample; Gaussian fittings of different diluted sample; Absorption spectra of Plastic bottle samples; Absorption spectra of sample after succussions.

\section{AUTHOR INFORMATION}

\section{Corresponding Author}

Chandra Sekhar Tiwary*

Metallurgical and Materials Engineering, Indian Institute of Technology Kharagpur, Kharagpur721302, India

E-mail: chandra.tiwary@metal.iitkgp.ac.in

\author{
ABBREVIATIONS \\ NPs, Nanoparticles; XRD, X-ray powder diffraction; TEM, Transmission electron microscopy; \\ FWHM, Full width half maximum;
}




\section{REFERENCES}

[1] Lohse, S. E.; Murphy, C. J. Applications of Colloidal Inorganic Nanoparticles: From Medicine to Energy, J. Am. Chem. Soc. 2012, 134, 15607-15620. dx.doi.org/10.1021/ja307589n

[2] Duchesne, L.; Gentili, D.; Franchini, M. C.; Fernig, D. G. Robust Ligand Shells for Biological Applications of Gold Nanoparticles, Langmuir 2008, 24, 13572-13580. 10.1021/la802876u

[3] Jain, P. K.; Lee, K. S.; El-Sayed, I. H.; El-Sayed, M. A. Calculated Absorption and Scattering Properties of Gold Nanoparticles of Different Size, Shape, and Composition: Applications in Biological Imaging and Biomedicine, J. Phys. Chem. B 2006, 110, 7238-7248, 10.1021/jp057170o

[4] Tsunoyama, H.; Sakurai, H.; Ichikuni, N.; Negishi, Y.; Tsukuda, T. Colloidal Gold Nanoparticles as Catalyst for Carbon-Carbon Bond Formation: Application to Aerobic Homocoupling of Phenylboronic Acid in Water, Langmuir 2004, 20, 26, 11293-11296, 2004 DOI: $10.1021 / \mathrm{la} 0478189$

[5] Jin, R.; Zeng, C.; Zhou, M.; Chen, Y. Atomically Precise Colloidal Metal Nanoclusters and Nanoparticles: Fundamentals and Opportunities, Chem. Rev. 2016, 116, 10346-10413 DOI: 10.1021/acs.chemrev.5b00703

[6] Chikramane, P. S.; Suresh, A. K.; Bellare, J. R.; Kane, S. G. Extreme homeopathic dilutions retain starting materials: A nanoparticulate perspective, Homeopathy, 2010, 99, 231-242. doi: 10.1016/j.homp.2010.05.006. PMID: 20970092

[7] Basu, A.; Temgire, M. K.; Suresh, A. K.; Bellare, J. R. Dilution-Induced Physico-Chemical Changes of Metal Oxide Nanoparticles Due to Homeopathic Preparation Steps of Trituration and Succussion, Homeopathy, 2020, 109, 65-78. doi: 10.1055/s-0039-1694720.

[8] Chikramane, P. S.; Suresh, A. K.; Kane, S. G.; Bellare, J. R. Metal Nanoparticle Induced Hormetic Activation: A Novel Mechanism of Homeopathic Medicines, Homeopathy, 2017, 106, 135-144. doi: 10.1016/j.homp.2017.06.002.

[9] Wassenhoven, M. V.; Goyens, M.; Capieaux, E.; Devos, P.; Dorfman, P. Nanoparticle Characterisation of Traditional Homeopathically Manufactured Cuprum Metallicum and Gelsemium Sempervirens Medicines and Controls, Homeopathy, 2019, 108, 73-74. doi: 10.1055/s-0038-1676304. Epub 2018 Dec 12. PMID: 30541166.

[10] Bhattacharyya, S. S.; Paul, S.; Khuda-Bukhsh, A. R. Encapsulated Plant Extract (Gelsemiumsempervirens) Poly (Lactide-Co-Glycolide) Nanoparticles Enhance Cellular 
Uptake and Increase Bioactivity in Vitro. Exp. Biol. Med., 2010, 235, 678-688. doi: 10.1258/ebm.2010.009338. PMID: 20511672.

[11] Nandy, P. A Review of Basic Research On Homoeopathy from A Physicist's Point of View, Indian J Res Homoeopathy, 2015, 9, 141-151. DOI: 10.4103/0974-7168.166372.

[12] Upadhyay, R. P.; Nayak, C. Homeopathy Emerging as Nanomedicine, Int J High Dilution Res. 2011, 10, 299-310.

[13] Rajendran, E. S. Homeopathy Seen as Personalised Nanomedicine, Homeopathy, 2019, 108, 66-70. doi: 10.1055/s-0038-1669988.

[14] Bell, I. R.; Schwartz, G. E. Adaptive Network Nanomedicine: An Integrated Model for Homeopathic Medicine, Front Biosci. 2013, 5, 685-708. doi: 10.2741/s400. PMID: 23277079.

[15] Bell, I. R.; Schwartz, G. E. Enhancement of Adaptive Biological Effects by Nanotechnology Preparation Methods in Homeopathic Medicines, Homeopathy 2015, 104, 123-38. doi: 10.1016/j.homp.2014.11.003.

[16] Homeopathic Pharamacopeia of India (HPI), https://abfh.org.br/wpcontent/uploads/2017/03/HPI-COMBIND-VOLUME-I-V

[17] Allen, Timothy Field, The encyclopedia of pure materiamedica: a record of the positive effects of drugs upon the healthy human organism, American Foundation for Homoeopathy. Mat. Med. Vol. II, 1. http://resource.nlm.nih.gov/64240040R.

[18] Gaspera, E. D.; Karg, M.; Baldauf, J.; Jasieniak, J.; Maggioni, G.; Martucci, A. Au Nanoparticle Monolayers Covered with Sol-Gel Oxide Thin Films: Optical and Morphological Study, Langmuir 2011, 27, 13739-13747. dx.doi.org/10.1021/la2032829. |

[19] Kumbhakar, P.; Biswas, S.; Kumbhakar, P. Observation of High Photocatalytic Activity by Tuning of Defects in Chemically Synthesized Ethylene Glycol Capped Znonanorods, Optik 2018, 154, 303-314. https://doi.org/10.1016/j.jileo.2017.10.039.

[20] Chikramane, P. S.; Kalita, D.; Suresh, A. K.; Kane, S. G.; Bellare, J. R. Why Extreme Dilutions Reach Non-Zero Asymptotes: A Nanoparticulate Hypothesis Based on Froth Flotation, Langmuir 2012, 28, 15864-15875. https://doi.org/10.1021/la303477s.

[21] Kelly, K. L.; Coronado, E.; Zhao, L. L.; Schatz, G. C. The Optical Properties of Metal Nanoparticles: The Influence of Size, Shape, and Dielectric Environment, J. Phys. Chem. B. 2003, 107, 668-677. https://doi.org/10.1021/jp026731y. 
[22] Feng, X. T.; Zhang, F.; Wang, Y. L.; Zhang, Y.; Yang, Y. Z.; Liu, X. G. Luminescent Carbon Quantum Dots with Highquantum Yield as A Single White Converter for white Light Emitting Diodes, Appl. Phys. Lett. 2015, 107, 213102. https://doi.org/10.1063/1.4936234.

[23] Pan, D.; Zhang, J.; Li, Z.; Wu, M. Hydrothermal Route for Cutting Graphene Sheets into Blue-Luminescent Graphene Quantum Dots, Adv. Mater. 2010, 22, 734-738. DOI: 10.1002/adma.200902825.

[24] Ilaslan, K.; Boyaci, I. H.; Topcu, A. Rapid Analysis of Glucose, Fructose and Sucrose Contents of Commercial Soft Drinks Using Raman Spectroscopy, Food Control, 2015, 48, 56-61. https://doi.org/10.1016/j.foodcont.2014.01.001.

[25] Yang, X.; Zhang, A.Y.; Wheeler, D. A.; Bond, T. C.; Gu, C.; Li, Y. Direct Molecule-Specific Glucose Detection by Raman Spectroscopy Based on Photonic Crystal Fiber. Anal Bioanal Chem. 2012, 402, 687-691. https://doi.org/10.1007/s00216-011-5575-1.

[26] Penkov, N. V. Temporal Dynamics of the Scattering Properties of Deionized Water. Phys. Wave Phen. 2020, 28, 135-139. https://doi.org/10.3103/S1541308X20020132.

[27] Ushikubo, F. Y.; Furukawa, T.; Nakagawa, R.; Enari, M.; Makino, Y.; Kawagoe, Y.; Shiina, T.; Oshita, S. Evidence of the Existence and the Stability of Nano-bubbles in Water, Colloids Surf. A. 2010, 361, 31-37. https://doi.org/10.1016/j.colsurfa.2010.03.005. 